\title{
Artelogie
}

Recherche sur les arts, le patrimoine et la littérature de l'Amérique latine

2 | 2012

Mexique : espace urbain et résistances artistiques et littéraires face à la « ville générique »

\section{La ville dans la littérature mexicaine, effritements, pouvoirs et mensonges}

Philippe Ollé-Laprune

\section{OpenEdition}

Journals

Édition électronique

URL : https://journals.openedition.org/artelogie/8032

DOI : 10.4000/artelogie.8032

ISSN : 2115-6395

Éditeur

Association ESCAL

Référence électronique

Philippe Ollé-Laprune, "La ville dans la littérature mexicaine, effritements, pouvoirs et mensonges », Artelogie [En ligne], 2 | 2012, mis en ligne le 21 janvier 2012, consulté le 07 janvier 2022. URL : http:// journals.openedition.org/artelogie/8032 ; DOI : https://doi.org/10.4000/artelogie.8032

Ce document a été généré automatiquement le 7 janvier 2022

Association ESCAL 


\title{
La ville dans la littérature mexicaine, effritements, pouvoirs et mensonges
}

\author{
Philippe Ollé-Laprune
}

1 La littérature mexicaine est comme la maison mexicaine, en général tournée vers l'intérieur. Elle regarde son propre environnement, poussée par la volonté d'en déchiffrer les secrets et les troubles. Comme ce fut le cas dans de nombreux pays, le passage d'un monde rural à un monde urbain caractérise cette société au XXème siècle ; au cours des années 50 les derniers feux de la campagne brillent encore dans des chefs d'oeuvre comme Al filo del agua de Agustín Yáñez ou chez Rulfo et Arreola. Avec La región más transparente, Carlos Fuentes impose Mexico comme espace incontournable de ses obsessions, lui offrant non seulement le rôle de scène vertigineuse mais aussi une présence qui fait de cette capitale un être vivant, un protagoniste de sa fiction. Sa richesse et sa complexité, le poids du passé si vivace à l'intérieur du présent, les strates sociales si distinctes mais qui se rejoignent aussi dans des valeurs communes : tout cela fait que Fuentes met à jour la complexité d'un lieu qui, jusque-là, se bornait à être l'espace du basculement d'une société rurale vers un univers citadin.

2 La ville est l'espace du secret et du mensonge. Cela se voit clairement quand on se penche sur le roman de la Révolution. Rappelons que ces dix années de tourmentes, de chevauchés éperdues, de batailles meurtrières et des fusillades incessantes ont lieu dans les régions, dans les campagnes. Mexico est, en général, épargné par ce mouvement; les habitants de la capitale n'auront pas à souffrir des massacres qui se multiplient dans le pays. Les livres de Rafael Múñoz relatent le coté trépidant des combats; la campagne est le lieu de la transparence, l'espace dans lequel le récit se borne à transcrire avec fidélité les luttes et les succès, ou les échecs. L'espace dans lequel la nature est reine ne saurait mentir. En revanche, les romans de Martín Luis Guzmán, plus ambitieux dans les formes aussi, racontent comment la Révolution est trahie quand les gens des villes la récupèrent. Le souffle est retombé, les cavalcades laissent place à l'organisation de la cité : les espoirs populaires sont détournés et la 
manipulation des populations commence. Peu importe la relation avec le réel, si l'écrivain tente de rendre compte de faits historiques ou non. L'essentiel est de voir combien la campagne reste comprise comme l'univers simple où la vérité est à portée de main, proche et disponible, et combien la capitale est le lieu de l'intrigue, du mystère, de l'opacité et du mensonge. Avec El águila y la serpiente et surtout La sombra del caudillo, Guzmán met le doigt sur ce qui deviendra une constante dans cette littérature : Mexico est le lieu dans lequel se concentrent les mystères de cette société avec son cortège de silences, de fabrication de vérités nouvelles et artificielles, de pouvoirs manipulateurs et de créations d'illusions que la vie se charge de démentir. Les villages et le monde rural continueront à exister dans la narration mexicaine (pensons à Rulfo ou Yáñez, ou plus proche de nous Gardea ou Toscana), mais l'utilisation de la ville, presque toujours la capitale, va servir à l'élaboration de récits qui maintiennent ces obsessions liées à la manipulation, aux jeux de miroir et aux mystères intrigants qui peuplent ces pages.

Quel que soit le pays, la construction de la ville répond au goût de l'être humain de se donner un refuge, un espace solide qui contrarie les impositions de la nature et regroupe des populations sur un espace réduit. Pour l'écrivain, l'observation des passions devient un exercice plus simple. Mais ce milieu qui appelle la fermeté et la solidité, qui est comme le lieu qui protège des méfaits de la nature, procure, au Mexique, une sensation distincte : le coté changeant, toujours en construction et en bouleversement que connaît l'espace urbain se traduit par une absence de repères personnels. La construction manque d'assise: elle fait partie du provisoire et n'appartient pas à l'ordre de l'immuable. L'histoire de chacun doit se plaquer sur l'histoire du lieu: seuls dans l'entourage résistent les monuments et les souvenirs intimes liés à une maison ou un espace qui font partie du passé, restent plus souvent présents dans le souvenir que dans la réalité. Le lieu qui devrait être l'espace du repère, comme cela est le cas pour la ville dans le monde occidental, est plus un espace de brouillage et d'effritement. Les signes que le passé nous adresse dans le paysage ont, à Mexico, un aspect officiel; le destin personnel ne peut trouver ses marques originales et doit se rattacher à un bâtiment historique, à un élément qui est légué par le pouvoir du passé.

L'autorité a aussi organisé cela dans le domaine de la réflexion: pendant longtemps la lecture du passé a été un exercice au service des valeurs défendues par ce pouvoir. Il n'y a pas de façon de trouver des marques hors de celles que l'on reçoit comme imposées. De là un goût certain pour un retour en arrière, par ces romans historiques qui mettent en évidence des racines, une identité, un sens dans le paysage qui entoure les Mexicains. Et tout cela fait sens pour des auteurs aussi divers que Fuentes dans Cristóbal Nonato, del Paso avec son Palinuro de México ou García Ponce avec sa Crónica de la Intervención. D'autres auteurs plus récents se livrent à cet exercice, cèdent à la tentation d'aller fouiller ce passé pour mieux se retrouver dans un cadre qui soit compréhensible. Carmen Boullosa, Enrique Serna ou Álvaro Uribe, ont chacun à leur façon écrit des fictions qui eurent lieu en des temps lointains, quand l'environnement rassurait par sa clarté, sa fermeté. Ces histoires parlent d'époques durant lesquelles l'échelle de la ville permettait de prendre ses marques, de se trouver une place, à l'inverse des temps modernes où la croissance du tissu urbain enferme chacun dans un anonymat d'où, semble-t-il, est exclue toute possibilité de se trouver une identité propre. 
Si bien des chroniqueurs ont analysé cela, comme le formidable et inévitable Carlos Monsiváis, les auteurs de fictions sont aussi nombreux à avoir observé cette condition du «Mexicain dans la grande ville». Le jeune héros de De perfil de José Agustín est balloté dans un espace qui le dépasse, qu'il ne maîtrise pas. Une des grandes réussites de ce roman initiatique vient justement de la manière dont l'auteur réussit à faire correspondre l'âge des grands questionnements et d'un certain flou intellectuel, l'adolescence, avec un espace géographique qui obéit à cette même logique, proche des mêmes sensations. L'entrée à l'Université, lieu reconnaissable et défini, le fait sortir du flou de la tâche urbaine, comme s'il s'agissait de l'entrée dans l'âge adulte.

L'autre grand contemporain qui observe avec attention sa ville et fait part de ses angoisses et son irrémédiable détresse, est José Emilio Pacheco. En effet, tant dans son œuvre poétique que dans ses textes narratifs, Mexico est le symbole de la souffrance, de la désintégration, de la perte inévitable de repères et de la catastrophe toujours à venir. Elle est l'image et le symbole des temps modernes où la dégradation permanente, lente ou brutale, du paysage, accompagne la détérioration de l'esprit humain tel qu'on le comprenait jusque-là. L'espace urbain est inquiétant et entretient un rapport au temps très particulier ; il est l'objet d'une vitalité surprenante et désespérante, d'une énergie qui fait qu'il meurt et renaît sans cesse, change de forme et évolue inexorablement. Pour Pacheco, la ville est l'espace de la confusion et de la douleur, le lieu où les contraires cohabitent et se répondent, où les souvenirs regardent plus du côté de l'imaginaire que des faits historiques. Si l'on veut porter une parole individuelle sur le passé, seule la reconstruction issue du chaos s'impose. Il commence son court récit Las batallas en el desierto, par ces mots : « Me acuerdo, no me acuerdo ». Etrange annonce qui est suivie d'une litanie de détails de la vie quotidienne qui cherche à trouver un espace aux souvenirs, à les définir contre un environnement qui ne cesse de dévorer les traces. Cette difficulté à trouver des limites apparaît souvent sous sa plume. Déjà dans les années 70, il développe le thème de la fatalité, de la ville qui disparaît sous les coups du temps qui passe et de la souffrance qui s'y accumule. Dans son poème México: vista aérea, il écrit :

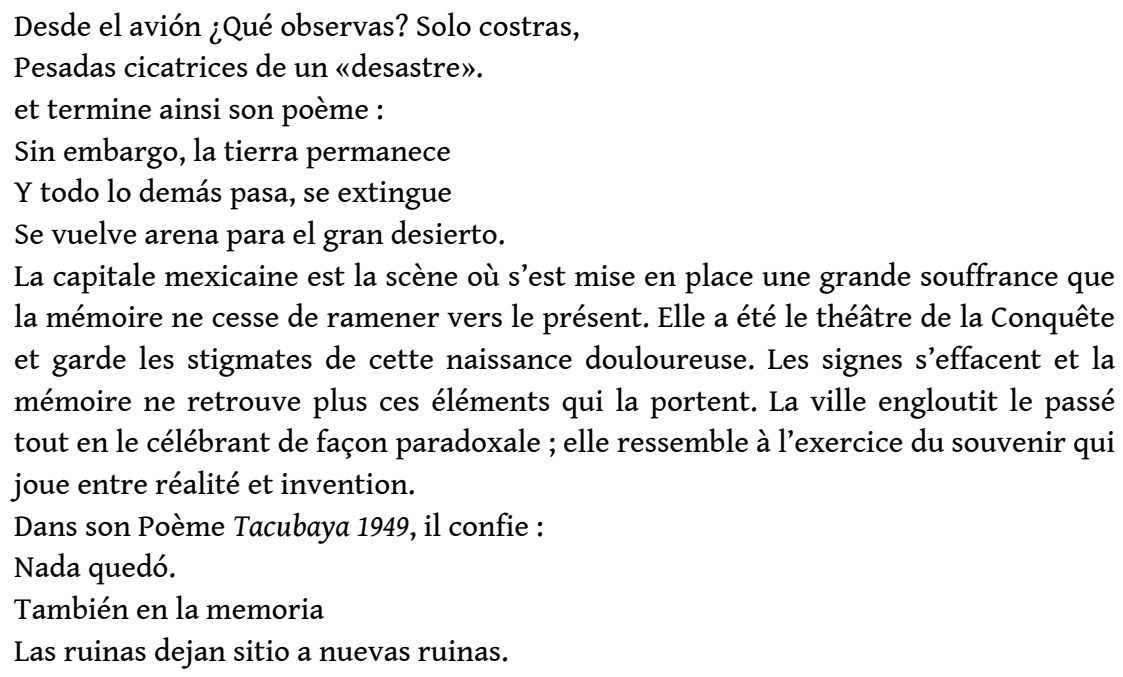

7 Il touche là un des thèmes qui va obséder ses pairs : la facilité avec laquelle l'ambiance et l'histoire mexicaine permettent de s'inventer un passé ou un présent, la manière dont la perte des marques cause un effritement de l'identité qui autorise toutes les créations artificielles pour remédier à ce chaos, et comment l'exercice du pouvoir, totalement urbain depuis longtemps, se nourrit de cette vacuité qui permet tous les 
abus. Pacheco le souligne encore quand il écrit un autre poème intitulé Capital del vacío où il insiste sur le caractère catastrophique et désastreux du lieu. L'histoire le rattrape avec le tremblement de terre de 1985 auquel il consacre un de ses textes les plus fameux : Las ruinas de México (Elegía de México). Là, plus qu'ailleurs, sa voix prend un ton dramatique pour dire combien la création de l'homme (la ville en ce cas) est vouée à la destruction et combien notre destin est d'en subir les terribles conséquences. Encore une fois l'espace urbain est plus une menace qu'un motif de tranquillité ; les remparts que semblent élever l'avancée de la ville ne sont qu'une illusion. La fatalité de l'histoire ramène inévitablement vers la destruction et la ruine.

8 La chronique est un domaine d'écriture particulièrement prisé au Mexique ; à la croisée du journalisme et de la littérature, elle donne vie à des remarques, des sensations et des réflexions qui accompagnent la vie des Mexicains. Le premier texte écrit qui émane de ce territoire est une chronique de la Conquête. Et cela va continuer au XXème siècle avec la figure marquante du chroniqueur, comme l'ont été Salvador Novo ou Carlos Monsiváis. Certains des écrivains de fiction qui pratiquent aussi l'écriture de ce genre de textes nous donnent les meilleures représentations de la ville et les clefs les plus efficaces pour saisir une réalité qui nous échappe souvent. Juan Villoro est sans conteste le mieux armé parmi les narrateurs mexicains contemporains pour nous offrir dans ses romans une image de la capitale, une grille qui permet de découvrir les énigmes du lieu et une somme d'images et de sensations qui disent combien cet environnement fait surgir des questionnements. Il a étudié la sociologie et porte un regard aigu sur cet univers, avec des points de vue originaux comme dans ce texte ${ }^{1}$ où il voit que la ville est le ciel du métro.

9 La grande réussite de ses trois romans vient de son talent à rendre compte de la complexité des émois et des questionnements que fait surgir ce lieu. Dans El disparo de Argón, son premier roman, l'allégorie de la ville est un hôpital, un espace où la manipulation et le mensonge sont les outils du pouvoir. Dès les premières lignes, l'auteur dit combien la sensation d'artificiel intervient dans le décor: "Un cielo cerrado, artificial». Et ce thème va hanter les pages des romans publiés jusqu'à aujourd'hui. Comment trouver une frontière entre l'artificiel et l'authentique dans un univers aussi trouble. Dans Materia dispuesta, le héros prend ses repères grâce aux seuls faits indéniables, réels : les tremblements de terre. La trame du récit se tend entre deux mouvements telluriques. Il vit en marge de la ville, dans un quartier inventé par l'écrivain; cette périphérie illustre à quel point ce personnage évolue au bord de l'authentique, perdu dans un monde qui invite malaisément à différencier ce qui est certain de ce qui est douteux.

Mexico est l'espace où se déploie cette œuvre qui embrasse avec humour, sans démagogie, le désir de dénoncer les faux semblants et les créations fallacieuses. Avec $E l$ testigo, Villoro boucle sa réflexion en faisant intervenir un héros qui revient après des années vécues à l'étranger; il a échappé à cette ville pendant des années et doit entreprendre un nouvel apprentissage des mystères du lieu. L'outil artificiel par excellence, centre du simulacre, est la télévision, dont ce personnage dispose pour reconstruire le passé. Il va travailler sur l'un des épisodes les plus occultés et les plus manipulés de l'histoire mexicaine, la Guerre des Cristeros, pour en faire une émission de télévision. Là encore, la reconstruction du passé est le symbole de l'imposition du factice. Et durant tout le livre, les choses ne sont pas ce qu'elles semblent être, les hommes de loi, les policiers, sont les plus corrompus. Des mythes nouveaux sont créés, 
telle cette espèce de secte qui glorifie le poète Lopez Velarde, comme si les temps modernes pouvaient pervertir les éléments les plus incontestables et les plus purs du passé, comme cela est ici le cas pour ce grand poète fondateur de la modernité au Mexique. En contrepoint de tous ces mensonges et ces tromperies, le vent du désert et le goût de la terre sont les seules certitudes qui restent au protagoniste pour sentir quelque chose de vrai, d'indéniable. Villoro nous transmet l'égarement que cette immense conurbation réserve à ceux qui y vivent. Mais au-delà de l'anecdote, c'est bien une interrogation sur la modernité qu'il nous lance, sur le malaise qui accompagne notre façon d'être au monde. Mexico lui sert pour ses desseins, son énormité la transforme en pointe extrême des multiples maux qui accablent notre temps.

11 Face à cette gigantesque tache urbaine, les écrivains sont saisis de vertige. La croissance de cet espace change la nature des rapports de chaque individu avec son environnement. Il y a comme un basculement, un changement dans l'essence même des échanges qui s'y produisent. Nous sommes cernés par les mensonges, les leurres et les constructions artificielles en tous genres. On s'enfonce dans Mexico comme on rentre dans un récit de fiction, sans repères et sans autre certitude que de savoir que l'on participe à la catastrophe douce et permanente qui s'impose sans frictions ni résistances.

\section{BIBLIOGRAPHIE}

AGUSTíN José, De perfil, Joaquín Mortiz, Mexico 1966.

DEL PASO Fernando, Palinuro de México, Joaquín Mortiz, Mexico, 1980.

FUENTES Carlos, La región más transparente, Fce, Mexico 1958.

FUENTES Carlos, Cristobal Nonato, Mexico 1987.

GARCIA PONCE Juan, Crónica de la Intervención, Conaculta, Mexico, 1992.

GUZMAN Martín Luís, El águila y la serpiente, Aguilar, Madrid, 1928.

GUZMAN Martín Luís, Las sombras del caudillo, Espasa-Calpe, Madrid 1929.

PACHECO José Emilio, Las batallas en el desierto, Joaquin Mortiz, Mexico1981.

PACHECO José Emilio, Tarde o temprano, Fce, Mexico 2000.

VILLORO Juan, El disparo de Argon, Alfaguara, Madrid 1991.

VILlORO Juan, Materia dispuesta, Alfaguara, Mexico 1996.

VILLORO Juan, El testigo, Anagrama, Barcelona, 2004.

YAÑEZ Agustín, Al filo del agua, Porrua, Mexico 1947. 


\section{NOTES}

1. La ciudad es el cielo del metro, La Torre, Revista de la Universidad de Puerto Rico, Vol. 4, $\mathrm{N}^{\circ} 12$, 1999.

\section{RÉSUMÉS}

Dans toute littérature, la présence de la ville s'accentue avec le temps. La société urbaine devient majoritaire au XXème siècle au Mexique. Mais une spécificité accompagne ce développement; alors que partout ailleurs, la ville est le symbole de la fermeté et de la solidité, Mexico rentre en littérature en présentant un caractère d'effritement permanent, de lieu catastrophique par excellence, qui ne protège en rien ses habitants. Lieu d'où sont absents les repères personnels (un comble pour un espace urbain), elle invite plus au chaos et au mensonge qu'à la transparence et à la raison. Cela est remarquable chez bon nombre d'écrivains contemporains, en particulier José Emilio Pacheco et Juan Villoro.

\section{INDEX}

Mots-clés : Mexico, José Emilio Pacheco, Juan Villoro

\section{AUTEUR}

\section{PHILIPPE OLLÉ-LAPRUNE}

Ecrivain et éditeur, Mexico D.F. 